\title{
Successful transplantation of peripheral blood stem cells mobilized by chemotherapy and a single dose of pegylated G-CSF in patients with multiple myeloma
}

\author{
U Steidl, R Fenk, I Bruns, F Neumann, M Kondakci, B Hoyer, T Gräf, U-P Rohr, S Bork, \\ R Kronenwett, R Haas and G Kobbe \\ Department of Hematology, Oncology and Clinical Immunology, Heinrich Heine University Duesseldorf, Duesseldorf, Germany
}

\begin{abstract}
Summary:
Following induction therapy and $4 \mathrm{~g} / \mathrm{m}^{2}$ cyclophosphamide, a single dose of $12 \mathrm{mg}$ polyethyleneglycolconjugated G-CSF (pegfilgrastim; $n=12$ ) or daily doses of unconjugated G-CSF $(8.5 \mu \mathrm{g} / \mathrm{kg} / \mathrm{day})(n=12)$ were administered to myeloma patients. Pegfilgrastim was associated with an earlier leukocyte recovery (12 vs 14 days) and peripheral blood CD34 + cell peak (12 vs 15 days). The peripheral blood $\mathrm{CD34}+$ cell peak was lower in the pegfilgrastim group (78 vs $111 / \mu \mathrm{l})$. Following highdose melphalan $\left(200 \mathrm{mg} / \mathrm{m}^{2}\right)$ and autografting, leukocyte and platelet reconstitution was similar in both groups and stable blood counts were observed 100 days post transplant. In summary, a single dose of pegfilgrastim after chemotherapy is capable of mobilizing a sufficient number of CD34 + cells for successful autografting with early engraftment and sustained hematological reconstitution in patients with myeloma. These data provide the basis for randomized studies evaluating the optimal dose and time of pegfilgrastim as well as long-term outcome in larger cohorts of patients.

Bone Marrow Transplantation (2005) 35, 33-36. doi:10.1038/sj.bmt.1704702
\end{abstract}

Published online 8 November 2004

Keywords: stem cell transplantation; stem cell mobilization; multiple myeloma; G-CSF; polyethylenglycole

High-dose therapy and autologous blood stem cell transplantation (ABSCT) are widely used in myeloma. ${ }^{1-4}$ Approximately $2.5 \times 10^{6} \mathrm{CD} 34+$ cells $/ \mathrm{kg}$ bodyweight provide rapid and sustained hematopoietic reconstitution after myeloablative therapy. ${ }^{5}$ Different methods have been used to mobilize hematopoietic stem and progenitor cells into the peripheral blood such as cytotoxic therapy alone, hematopoietic growth factors, or a combination of both. ${ }^{6-8}$

Correspondence: Dr U Steidl, Beth Israel Deaconess Medical Center, Harvard Institutes of Medicine, Room 907, 77 Avenue Louis Pasteur, Boston, MA 02115, USA; E-mail: usteidl@bidmc.harvard.edu

Received 10 April 2004; accepted 5 July 2004

Published online 8 November 2004
Recently, a polyethylene glycol (PEG)-conjugated form of G-CSF has been introduced (pegfilgrastim, Neulasta ${ }^{\mathrm{TM}}$ ). ${ }^{9}$ In this formulation, filgrastim is bound covalently to a $20 \mathrm{kDa}$ PEG molecule which increases the serum half-life of G-CSF due to decreased renal elimination. ${ }^{10}$ As a result, therapeutic serum levels of G-CSF are maintained over a period of about 2 weeks following the subcutaneous injection of a single dose of pegfilgrastim. In patients with lymphoma, pegfilgrastim is as effective as filgrastim in shortening the time of neutropenia after cytotoxic chemotherapy. The spectrum of side effects is similar to that encountered in patients who have received filgrastim. ${ }^{1-13}$ However, systematic data on the ability of pegfilgrastim to mobilize stem cells after chemotherapy as well as on the engraftment potential of pegfilgrastim-mobilized CD34+ cells are lacking.

\section{Patients and methods}

After having obtained written informed consent, 24 patients with newly diagnosed myeloma were studied. Treatment protocols were approved by the local ethics committee of the Heinrich Heine University Duesseldorf. Patients were treated with an induction therapy consisting of 1-6 cycles of either $4 \times 10 \mathrm{mg} / \mathrm{m}^{2}$ idarubicin p.o. in combination with $4 \times 20 \mathrm{mg} / \mathrm{m}^{2}$ dexamethasone p.o. (ID), or $2 \mathrm{mg}$ vincristine i.v. combined with $36 \mathrm{mg} / \mathrm{m}^{2}$ doxorubicine i.v. given as a $96 \mathrm{~h}$ infusion and $4 \times 40 \mathrm{mg}$ dexamethasone p.o. (VAD). Following induction therapy, all patients received a total dose of $4 \mathrm{~g} / \mathrm{m}^{2}$ cyclophosphamide administered on two consecutive days.

A cohort of 12 consecutive patients who are referred to as the 'pegfilgrastim group' received a single dose of $12 \mathrm{mg}$ pegfilgrastim on day 4 (median, range 3-6) after cyclophosphamide. Mobilization kinetics, apheresis results and transplantation outcomes were retrospectively compared with 12 patients with similar characteristics, who had received a median daily dose of $8.5 \mu \mathrm{g}$ (range 4.6-11.9 $\mu \mathrm{g}$ )/ $\mathrm{kg}$ bodyweight of unconjugated G-CSF (11 filgrastim, one lenograstim) subcutaneously starting 5 days (median, range 2-7) after cyclophosphamide. The growth factor was given daily until a sufficient number of CD34 + cells could be 
harvested. In the following, these patients will be referred to as the 'G-CSF group'.

Patients of the pegfilgrastim group did not show any significant differences with regard to age, gender, body weight, subtype of the myeloma, stage, or induction therapy, when compared to the G-CSF group. The patients' characteristics are summarized in Table 1.

All patients were followed with routine physical and blood examinations in our outpatient clinic on a regular basis depending on their general condition. When the patients had gone through the nadir of the white blood cell count (WBC), the CD34 + cells in the peripheral blood were measured as early as the $\mathrm{WBC}$ rose above $1 \times 10^{9} / 1$. The concentration of $\mathrm{CD} 34+$ cells was determined according to the International Society of Hematotherapy and Graft Engineering (ISHAGE) guidelines. ${ }^{14} \mathrm{~A}$ leukapheresis was performed 1 day after CD34 + cells exceeded a level of $10 \mu \mathrm{l}$ in the peripheral blood, and continued until at least $4 \times 10^{6} \mathrm{CD} 34+$ cells per kilogram bodyweight were collected. After successful mobilization and leukapheresis, peripheral blood stem cells were cryopreserved in liquid nitrogen and checked for viability and clonogenic growth before transplantation.

Patients were re-evaluated and received a high-dose therapy with a total dose of $200 \mathrm{mg} / \mathrm{m}^{2}$ melphalan administered on two consecutive days, followed by autologous transplantation of at least $2 \times 10^{6} \mathrm{CD} 34+$ cells per kilogram bodyweight. All patients received a standard supportive treatment including antibiotic, antimycotic and antiviral therapy. No growth factors were given after transplantation.

Student's $t$-test was used to compare the pegfilgrastim group with the G-CSF group regarding mobilization, leukapheresis and transplantation characteristics. $P$-values smaller than 0.05 are indicated (Tables 1-4).

\section{Results}

\section{Mobilization}

Following the administration of cyclophosphamide recovery of leukocytes to counts of more than $1 \times 10^{9} / 1$ was observed after 12 days (median, range 7-14 days) in patients of the pegfilgrastim group and after 14 days (median, range 11-15) in patients of the G-CSF group
$(P=0.05)$. There were no differences with regard to the time needed for platelet recovery to counts of greater than 20000 or $50000 / \mu 1$ respectively (Table 2 ). The maximum number of CD34 + cells in the peripheral blood was noted on day 12 (median, range 11-16) in patients of the pegfilgrastim group. This was significantly earlier in comparison to patients of the G-CSF group in whom the median peak was observed on day 15 (range 12-18). On the other hand, the median maximum CD34 + cell count in the peripheral blood was $78 / \mu 1$ (range $20-1055 / \mu$ ) in the pegfilgrastim group and $111 / \mu$ l (range 28-760) in the GCSF group. This difference was not statistically significant $(P=0.81)$.

\section{Leukapheresis}

In line with the earlier appearance of the peripheral $\mathrm{CD} 34+$ cell peak, the first apheresis in the pegfilgrastim group was performed 2 days earlier in comparison to the GCSF group (median day 13 vs 15, range 11-15 vs 12-18, $P=0.01$, Table 3 ). Within the G-CSF group, one apheresis procedure was sufficient to obtain at least $4 \times 10^{6} \mathrm{CD} 34+$ cells/ $\mathrm{kg}$ bodyweight in all patients. The same was true for 11 out of 12 patients in the pegfilgrastim group, whereas one patient needed a second apheresis to obtain an amount of CD34 + cells above this threshold. The total number of collected $\mathrm{CD} 34+$ cells $/ \mathrm{kg}$ bodyweight was $7.4 \times 10^{6}$ (median, range 4.9-38.0 $\times 10^{6}$ ) in the pegfilgrastim group and $10.8 \times 10^{6}$ (median, range 5.0-87 $\left.\times 10^{6}\right)$ in the G-CSF

Table 1 Patient characteristics

\begin{tabular}{lcc}
\hline & Pegfilgrastim group & G-CSF group \\
\hline Number of patients & 12 & 12 \\
Age & 51 years $(41-65)$ & 51 years $(32-62)$ \\
Gender (m/f) & $9 / 3$ & $7 / 5$ \\
Bodyweight & $78 \mathrm{~kg}(66-107)$ & $77 \mathrm{~kg}(51-97)$ \\
IgG, A, D & $7,3,0$ & $10,1,1$ \\
LC & 2 & 0 \\
Stage II, III & 4,8 & 4,8 \\
Stage A, B & 8,4 & 10,2 \\
Induction therapy (cycles) & $4(1-6)$ ID/VAD & $3(1-5) \mathrm{ID} / \mathrm{VAD}$ \\
Radiotherapy (total) & $4(33 \%)$ & $6(50 \%)$ \\
Extensive radiotherapy $^{\mathrm{a}}$ & $1(8 \%)$ & $3(25 \%)$ \\
\hline
\end{tabular}

${ }^{\text {a }}$ Patients with radiotherapy of seven or more vertebra and/or pelvis.

Table 2 Mobilization data following $4 \mathrm{~g} / \mathrm{m}^{2}$ cyclophosphamide

\begin{tabular}{lcc}
\hline & Pegfilgrastim group & G-CSF group \\
\hline Cytokine dose (total** & $12 \mathrm{mg}$ & $7.5 \mathrm{mg}(5.4-10.2)$ \\
Cytokine dose (calculated) & & $8.5 \mu \mathrm{g} / \mathrm{kg} / \mathrm{day}(7.0-13.3)$ \\
Number of applications* & $11(8-15)$ & $5(2-7)$ \\
First day of cytokine application & $4(3-6)$ & 14 days $(11-15)$ \\
Leukocyte reconstitution to $>1 \times 10^{9} / l^{\#}$ & 12 days $(7-14)$ & 12 days $(10-18)$ \\
Platelet reconstitution to $>20 \times 10^{9} / 1$ & 11 days $(10-15)$ & 14 days $(10-23)$ \\
Platelet reconstitution to $>50 \times 10^{9} / 1$ & 13 days $(10-17)$ & $15(12-18)$ \\
Day of peripheral blood CD34+ count maximum* & $12(11-16)$ & $111 / \mu \mathrm{l}(28-760)$ \\
Maximal peripheral blood CD34 + cell count & $78 / \mu \mathrm{l}(20-1055)$ & \\
\hline
\end{tabular}

$* P<0.05$.

${ }^{\#} P=0.05$. 


\begin{tabular}{lcc}
\hline & Pegfilgrastim group & G-CSF group \\
\hline Day of $1^{\text {st }}$ apheresis* & $13(11-15)$ & $15(12-18)$ \\
Number of aphereses & $1(1-2)$ & 1 \\
Processed volume & $12.21(5.6-37.1)$ & $14.91(8.8-27.1)$ \\
CD34 + cells/kg bodyweight & $7.4 \times 10^{6} / \mathrm{kg}(4.9-38.0)$ & $10.8 \times 10^{6} / \mathrm{kg}(5.0-87.0)$ \\
CD34 + cells/kg bodyweight and volume & $558 / \mathrm{kg} \mathrm{ml} \mathrm{(132-4488)}$ & $868 / \mathrm{kg} \mathrm{ml}(199-9622)$ \\
Mobilization failures & 0 & 0 \\
\hline
\end{tabular}

$* P<0.05$

Table 4 Transplantation data following high-dose therapy with melphalan $\left(200 \mathrm{mg} / \mathrm{m}^{2}\right)$ and autografting

\begin{tabular}{lcc}
\hline & Pegfilgrastim group & G-CSF group \\
\hline Transplanted patients & 8 & 12 \\
Transplanted CD $34+$ cells & $3.4 \times 10^{6} / \mathrm{kg}(2.5-19.0)$ & $7.5 \times 10^{6} / \mathrm{kg}(2.1-29.0)$ \\
Leukocyte reconstitution to $>1 \times 10^{9} / 1$ & 16 days $(13-24)$ & 15 days $(12-22)$ \\
Platelet reconstitution to $>20 \times 10^{9} / 1$ & 12 days $(10-26)$ & 12 days $(10-24)$ \\
Platelet reconstitution to $>50 \times 10^{9} / 1$ & 13 days $(10-39)$ & 13 days $(11-26)$ \\
Leukocyte count on day $100\left(10^{9} / 1\right)$ & $4.4(3.0-8.2)$ & $4.3(2.3-8.3)$ \\
Hemoglobin level on day $100(\mathrm{~g} / \mathrm{dl})$ & $12.6(10.6-14.0)$ & $12.3(10.6-14.9)$ \\
Platelet count on day $100\left(10^{9} / 1\right)$ & $155(72-258)$ & $147(64-291)$ \\
\hline
\end{tabular}

a

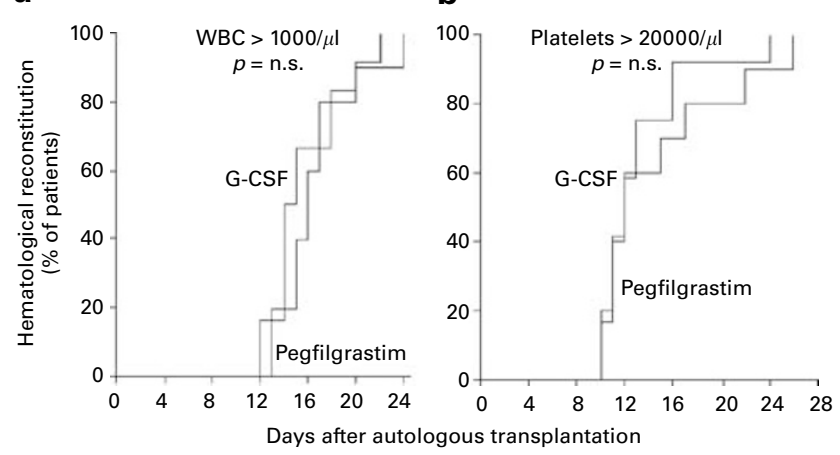

Figure 1 Hematological reconstitution following high-dose therapy with $200 \mathrm{mg} / \mathrm{m}^{2}$ melphalan and autologous transplantation of blood stem cells mobilized by chemotherapy plus single-dose pegfilgrastim or chemotherapy plus daily unconjugated G-CSF. (a) Leukocyte reconstitution $(>1000 / \mu \mathrm{l})$. (b) Platelet reconstitution $(>20000 / \mu \mathrm{l})$.

group (not significant, $P=0.26$ ). When we calculated the collected number of $\mathrm{CD} 34+$ cells $/ \mathrm{kg}$ bodyweight $/ 1$ processed volume, the difference was less significant $(P=0.60)$.

\section{High-dose therapy and autografting}

At the time of writing, each of the 12 patients from the GCSF group and eight patients of the pegfilgrastim group had a minimal follow-up of 100 days after high-dose cytotoxic therapy, which consisted of $200 \mathrm{mg} / \mathrm{m}^{2}$ of melphalan. The autografts used for transplantation contained a minimum of $2 \times 10^{6} \mathrm{CD} 34+$ cells. Patients of the pegfilgrastim group received a median of $3.4 \times 10^{6} \mathrm{CD} 34+$ cells per $\mathrm{kg}$ bodyweight (range $2.5-19.0 \times 10^{6}$ ) in comparison to $7.5 \times 10^{6}$ (range $\left.2.1-29.0 \times 10^{6}\right) \mathrm{CD} 34+$ cells $/ \mathrm{kg}$ bodyweight in patients of the G-CSF group (NS, $P=0.14$ ). Despite the lower number of CD34 + cells transplanted, there were no significant differences with regard to the time needed for hematological reconstitution. Leukocyte recovery was achieved after 16 days (median, range 13-24 days) in the pegfilgrastim group and after 15 days (median, range 12-22 days) in the G-CSF group (NS, $P=0.51$ ) (Figure 1a). Independence from platelet transfusions (platelets $>20000 /$ $\mu \mathrm{l})$ was observed after 12 days in both the pegfilgrastim and the G-CSF group (Figure 1b). Similarly, reconstitution of platelets to counts of more than $50000 / \mu$ l was achieved after a median of 13 days in both groups. All patients had stable blood counts 100 days after autologous transplantation (Table 4).

\section{Discussion}

Our data show that a single dose of pegfilgrastim following cytotoxic chemotherapy is capable of mobilizing sufficient numbers of peripheral blood stem cells for successful autografting after high-dose therapy in patients with myeloma. We chose a relatively high dose of $12 \mathrm{mg}$, which is twice the recommended dose to shorten neutropenia after conventional chemotherapy. A single dose of pegylated GCSF increases convenience and improves compliance when compared to the daily application of G-CSF from a practical point of view. ${ }^{15}$

The use of pegfilgrastim was associated with a reduction of 2 days for the time needed to reach a leukocyte count of more than $1 \times 10^{9} / 1$ and the peripheral blood CD34 + peak in comparison to daily G-CSF. As a result, the first leukapheresis was performed 2 days earlier compared with the patients who had received unconjugated G-CSF. The reason for this phenomenon might be related to the continuously high serum level of G-CSF maintained by pegfilgrastim, possibly providing a more efficient stimulus for the CD34+ hematopoietic stem and progenitor cells 
than the pulsatile way of action of daily G-CSF injections. However, these findings may also be related to the small patient number in this feasibility study and larger randomized trials are required to confirm this preliminary observation.

On the other hand, peak levels of CD34 + cells were lower in the pegfilgrastim group than in the G-CSF group, even though the difference was not significant. This was associated with a smaller number of CD34+ cells harvested $/ \mathrm{kg}$ bodyweight.

The pharmacokinetics of pegfilgrastim could explain the early appearance of the CD34 + cell peak and the lower number of $\mathrm{CD} 34+$ cells in the peripheral blood. As shown by pharmacokinetic studies in neutropenic patients, plasma G-CSF levels rise quickly after subcutaneous administration of pegfilgrastim and remain more or less stable due to impaired renal elimination. ${ }^{10,16-18}$ When the number of granulocytes increases, pegfilgrastim is eliminated by cellular uptake via the G-CSF receptor and intracellular degradation, as well as by cleavage through neutrophil elastase. ${ }^{19,20}$ Thus, the rise of leukocytes leads to a rapid clearance of G-CSF in the pegfilgrastim group, which then may result in less effective mobilization of CD34 + cells. The self-limiting effect of pegfilgrastim may not be relevant in shortening the duration of neutropenia, but may be so for effective stem cell mobilization. In order to utilize the pharmacokinetic advantages of pegylated G-CSF as well as those of unconjugated G-CSF for effective stem cell mobilization, a combination might be worthwhile.

Following high-dose therapy with $200 \mathrm{mg} / \mathrm{m}^{2}$ melphalan and autografting the time needed for leukocyte and platelet recovery was similar in both patient groups. There were no graft failures and adequate blood counts were achieved on day 100 post transplantation. Pegfilgrastim following chemotherapy apparently mobilizes CD34 + cells, which provide early engraftment and sustained hematological reconstitution.

In conclusion, mobilization, harvesting and autografting of pegfilgrastim-mobilized peripheral blood stem cells could be successfully achieved in patients with myeloma. Further studies are needed to address optimal dose and schedule of pegfilgrastim administration.

\section{Acknowledgements}

We thank Anke Boeckmann, Hildegard Gaussmann and Elke Rosenbaum-Koenig for expert technical support. This work was supported by Leukaemie Liga e.V., Duesseldorf.

\section{References}

1 Child JA, Morgan GJ, Davies FE et al. High-dose chemotherapy with hematopoietic stem-cell rescue for multiple myeloma. $N$ Engl J Med 2003; 348: 1875-1883.

2 Attal M, Harousseau JL, Stoppa AM et al. A prospective, randomized trial of autologous bone marrow transplantation and chemotherapy in multiple myeloma. Intergroupe Francais du Myelome. N Engl J Med 1996; 335: 91-97.
3 Badros A, Barlogie B, Siegel E et al. Autologous stem cell transplantation in elderly multiple myeloma patients over the age of 70 years. Br J Haematol 2001; 114: 600-607.

4 Attal M, Harousseau JL, Facon T et al. Single versus double autologous stem-cell transplantation for multiple myeloma. $N$ Engl $J$ Med 2003; 349: 2495-2502.

5 Haas R, Witt B, Mohle R et al. Sustained long-term hematopoiesis after myeloablative therapy with peripheral blood progenitor cell support. Blood 1995; 85: 3754-3761.

6 Haas R, Hohaus S, Goldschmidt $\mathrm{H}$ et al. Hematopoietic growth factors for the mobilization of peripheral blood stem cells. J Hematother 1993; 2: 357-359.

7 Kobbe G, Sohngen D, Bauser U et al. Factors influencing G-CSF-mediated mobilization of hematopoietic progenitor cells during steady-state hematopoiesis in patients with malignant lymphoma and multiple myeloma. Ann Hematol 1999; 78: 456-462.

8 Goldschmidt H, Hegenbart U, Haas R et al. Mobilization of peripheral blood progenitor cells with high-dose cyclophosphamide $\left(4\right.$ or $7 \mathrm{~g} / \mathrm{m}^{2}$ ) and granulocyte colony-stimulating factor in patients with multiple myeloma. Bone Marrow Transplant. 1996; 17: 691-697.

9 Curran MP, Goa KL. Pegfilgrastim. Drugs 2002; 62: $1207-1213$.

10 Zamboni WC. Pharmacokinetics of pegfilgrastim. Pharmacotherapy 2003; 23: 9S-14S.

11 Vose JM, Crump M, Lazarus $\mathrm{H}$ et al. Randomized, multicenter, open-label study of pegfilgrastim compared with daily filgrastim after chemotherapy for lymphoma. J Clin Oncol 2003; 21: 514-519.

12 George S, Yunus F, Case D et al. Fixed-dose pegfilgrastim is safe and allows neutrophil recovery in patients with nonHodgkin's lymphoma. Leuk Lymphoma 2003; 44: 1691-1696.

13 Grigg A, Solal-Celigny P, Hoskin $\mathrm{P}$ et al. Open-label, randomized study of pegfilgrastim $v s$ daily filgrastim as an adjunct to chemotherapy in elderly patients with nonHodgkin's lymphoma. Leuk Lymphoma 2003; 44: 1503-1508.

14 Sutherland DR, Anderson L, Keeney M et al. The ISHAGE guidelines for CD34 + cell determination by flow cytometry. International Society of Hematotherapy and Graft Engineering. J Hematother 1996; 5: 213-226.

15 Beveridge RA, Rifkin RM, Moleski RJ et al. Impact of longacting growth factors on practice dynamics and patient satisfaction. Pharmacotherapy 2003; 23: 101S-109S.

16 Johnston E, Crawford J, Blackwell S et al. Randomized, doseescalation study of $\mathrm{SD} / 01$ compared with daily filgrastim in patients receiving chemotherapy. J Clin Oncol 2000; 18: 25222528.

17 Crawford J. Pegfilgrastim administered once per cycle reduces incidence of chemotherapy-induced neutropenia. Drugs 2002; 62 (Suppl 1): 89-98.

18 Crawford J. Safety and efficacy of pegfilgrastim in patients receiving myelosuppressive chemotherapy. Pharmacotherapy 2003; 23: 15S-19S

19 El Ouriaghli F, Fujiwara H, Melenhorst JJ et al. Neutrophil elastase enzymatically antagonizes the in vitro action of GCSF: implications for the regulation of granulopoiesis. Blood 2003; 101: 1752-1758.

20 Hunter MG, Druhan LJ, Massullo PR et al. Proteolytic cleavage of granulocyte colony-stimulating factor and its receptor by neutrophil elastase induces growth inhibition and decreased cell surface expression of the granulocyte colony-stimulating factor receptor. Am J Hematol 2003; 74: 149-155. 